\title{
ON THE STRUCTURE OF SEMI-NORMAL OPERATORS ${ }^{1}$
}

BY C. R. PUTNAM

Communicated by P. R. Halmos, July 7, 1963

1. Preliminaries. Only bounded operators on a Hilbert space $\mathfrak{S}$ of elements $x$ will be considered. If $A$ is self-adjoint with the spectral resolution

(1) $A=\int \lambda d E(\lambda)$, and if $\mathfrak{S}_{a}=\mathfrak{S}_{a}(A)$ denotes the set of elements $x$ for which $\|E(\lambda) x\|^{2}$ is an absolutely continuous function of $\lambda$, then $\mathfrak{S}_{a}$ is a subspace; cf. $\left[2\right.$, p. 240], [3, p. 436] and [6, p. 104]. If $\mathfrak{S}_{\mathfrak{Y}}=\mathfrak{S}_{a}$, then $A$ is called absolutely continuous. The one-dimensional Lebesgue measure of the spectrum of a self-adjoint operator $A$ will be denoted by meas $\operatorname{sp}(A)$.

An operator $T$ on $\mathfrak{S}$ is called semi-normal if

(2) $T T^{*}-T^{*} T \equiv D \geqq 0$ or $D \leqq 0$.

There will be proved the following result concerning such an operator.

2. Theorem. If $T$ satisfies (2) and if $\mathfrak{M}=\mathfrak{M}_{T}$ is the smallest subspace of $\mathfrak{S}$ reducing $T$ and containing the range of $D$, then

(3) $T+T^{*}$ is absolutely continuous on $\mathfrak{M}$, and, if $\mathfrak{M}^{\perp}$ denotes the orthogonal complement of $\mathfrak{M}$ (so that $\mathfrak{M}^{\perp}$ also reduces $T$ ), then

(4) $T$ is normal on $\mathfrak{M}^{\perp}$.

In addition,

(5) $2 \pi\|D\| \leqq\left\|T-T^{*}\right\|$ meas $\operatorname{sp}\left(T+T^{*}\right)$,

and the inequality (5) is optimal in the sense that there exist examples with $D \neq 0$ for which (5) becomes an equality.

As a consequence, if $T$ is semi-normal but not normal, then $\mathfrak{S}_{a}\left(T+T^{*}\right) \neq 0$, a result which can also be concluded from [4, Corollary 3, p. 1029], where the symbol " $<$ " should be replaced by " $\neq$. ." (This same Corollary, incidentally, also implies the result proved by Andô [1] that a completely continuous semi-normal operator $T$ must be normal. In fact, if $T$ is completely continous, so also are $T^{*}$ and $T+T^{*}$. But the spectrum of $T+T^{*}$ clearly must be of measure zero.)

If $\theta$ is real and $T(\theta)=e^{i \theta} T$, then (2) is unchanged if $T$ is replaced by $T(\theta)$. Also, it is clear that the set $\mathfrak{M}_{T(\theta)}$ is independent of $\theta$. It follows that (3), (4) and (5) remain valid if, in each instance, $T$ is

${ }_{1}^{1}$ This work was supported by the National Science Foundation research grant NSF-G18915. 
replaced by $T(\theta)$. In particular then, relations (3) and (5) become assertions concerning the absolute continuity and spectra of both the real and the imaginary parts of a semi-normal operator $T$.

The proof of the Theorem will depend upon results proved in [5] and which will be stated here, in a form convenient for application, as a

Lemma. Let $H$ and $J$ be self-adjoint operators and suppose that

(6) $H J-J H=i C$, where $C \geqq 0$ or $C \leqq 0$. Then,

(7) $\mathfrak{i C} \mathfrak{S}_{a}(H)$, where $\mathfrak{R}$ denotes the smallest subspace reducing both $H$ and $J$ and also containing the range of $C$. Furthermore,

(8) $\pi\|C\| \leqq\|J\|$ meas $\operatorname{sp}(H)$.

It is clear from the symmetry of the condition (6) that (7) and (8) remain true if $H$ and $J$ are interchanged.

3. Proof of the Theorem. Let $T$ be represented as

(9) $T=H+i J$, where $H=\left(T+T^{*}\right) / 2$ and $J=\left(T-T^{*}\right) / 2 i$, so that (2) and (6) are equivalent by virtue of (9) and

(10) $D=2 C$.

It is clear that the space $\mathbb{R}$ of the Lemma must then coincide with the space $\mathfrak{M}$ of the Theorem. Relations (3) and (5) now follow respectively from (7) and (8), while relation (4) is a consequence of the fact that $\mathfrak{M}^{\perp}$ is contained in the null space of $D$. An example involving finite interval Hilbert transforms was given in [5] for which the hypothesis of the Lemma is fulfilled and for which (8) becomes an equality (with $C \neq 0$ ). This result in turn yields, by virtue of (9) and (10), an example in which equality holds in (5) and $D \neq 0$.

\section{REFERENCES}

1. T. Andô, On hyponormal operators, Proc. Amer. Math. Soc. 14 (1963), 290-291.

2. T. Kato, On finite-dimensional perturbations of self-adjoint operators, J. Math. Soc. Japan 9 (1957), 239-249.

3. S. T. Kuroda, On the existence and the unitary property of the scattering operator, Nuovo Cimento (10) 12 (1959), 431-454.

4. C. R. Putnam, On commutators and Jacobi matrices, Proc. Amer. Math. Soc. 6 (1956), 1026-1030.

5. - Commutators, absolutely continuous spectra, and singular integral operators (to appear).

6. P. R. Halmos, Introduction to Hilbert space, Chelsea, New York, 1951.

Purdue University 